\title{
Actitudes de los jóvenes ante situaciones de agravio en el entorno familiar: su relación con el género, la creencia religiosa y el clima social familiar*
}

\author{
Attitudes of Young People in Front of Offense \\ Situations in Family Context: its Relation to Gender, \\ Religious Belief and the Family Social Climate
}

Recibido: 13 de junio de 2012 | Revisado: 9 de mayo de 2013 | Aceptado: 12 de abril de 2014

\author{
MARÍA José CANTERo LóPEZ ** \\ PAZ Viguer Seguí **** \\ Rocío DOMÍNGUEZ BARRAGÁN ***** \\ Universidad de Valencia, España
}

doi:10.11144/Javeriana.upsy14-3.ajsa

Para citar este artículo: Cantero, M. J., Viguer, P., \& Domínguez, R. (2015). Actitudes de los jóvenes ante situaciones de agravio en el entorno familiar: su relación con el género, la creencia religiosa y el clima social familiar. Universitas Psychologica, 14(3), 1091-1106. http://dx.doi.org/10.11144/Javeriana. upsy14-3.ajsa

* Artículo original resultado de investigación

** Profesora Titular de Universidad. Departamento de Psicología Evolutiva y de la Educación de la Universidad de Valencia. Correo electrónico: maria.j.cantero@uv.es

*** Profesora Titular de Universidad. Departamento de Psicología Evolutiva y de la Educación de la Universidad de Valencia. Correo electrónico: paz. viguer@uv.es

****** Investigadora. Departamento de Psicología Evolutiva y de la Educación de la Universidad de Valencia. Correo electrónico: rociodominguezb@hotmail. com

\section{RESUMEN}

Los objetivos de este trabajo son, por una parte, analizar las actitudes de los jóvenes ante las situaciones de agravio que se producen en el contexto familiar en función del género, la creencia religiosa y el clima social familiar y, por otra, estudiar el poder predictivo de estas variables sobre las actitudes de agravio. Los participantes fueron 230 jóvenes (media de edad $=21.2$ ), 130 mujeres y 100 hombres, quienes cumplimentaron el cuestionario CASA-F (Moreno \& Pereyra, 2000) y la escala de Clima Social Familiar (Moos, Moos, \& Trickett, 1995). Se obtuvieron diferencias en las actitudes ante el agravio en función del género y la creencia religiosa y una relación significativa entre las actitudes prosociales ante el agravio y el clima social familiar. Por último, los análisis de regresión múltiple confirmaron la capacidad predictiva del género, la creencia religiosa y el clima social familiar sobre las actitudes prosociales de perdón y explicación.

Palabras clave

actitudes; ofensa; clima social familiar; creencia religiosa; perdón

\section{A B S T R A C T}

The objectives of this study are to analyze the attitudes of young people to offenders situations in family context according to gender, religious belief and family social climate and, to study the predictive power of those variables on attitudes toward offenders. Participants were 230 young (mean age $=21.2), 130$ women and 100 men who completed the CASA-F Questionnaire (Moreno \& Pereyra, 2000) and the Family Social Climate Scale (Moss, Moss, \& Trickett, 1995). We obtained differences in attitude towards offenders in terms of gender and religious belief and a significant relation between prosocial attitudes towards offenders and family social climate. Finally, multiple regression analysis confirmed the predictive ability of gender, religious belief and the family social climate on prosocial attitudes of forgiveness and explanation.

Keywords

attitudes; offenders; family social climate; religious beliefs; forgiveness 


\section{Introducción}

Las competencias y habilidades necesarias para resolver las situaciones interpersonales conflictivas se adquieren, básicamente, en el contexto de las relaciones afectivas con personas significativas, principalmente, en las relaciones familiares (Lafuente \& Cantero, 2010). Es en este contexto de las relaciones afectivas más cercanas, en el que se expresan e intercambian emociones y sentimientos, se comparten experiencias y vivencias, se presentan situaciones difíciles y se generan diferencias y encuentros que en algunos casos dan lugar a ofensas (Mikulincer \& Shaver, 2005; Pietromonaco, Greenwood, \& Feldman, 2004). El estudio de las relaciones afectivas y los conflictos asociados a ellas exige pues analizar las actitudes con las que nos enfrentamos a las ofensas.

De acuerdo con la definición del Diccionario de la Real Academia Española, definimos el agravio o la ofensa como 'la acción y efecto de ofender, humillar o herir el amor propio o la dignidad de alguien, o ponerlo en evidencia con palabras o con hechos'. La ofensa puede conducir a un punto crítico del vínculo interpersonal. Hay que tener en cuenta que la ofensa siempre está inserta dentro de un contexto, transcurre en un espacio y un tiempo determinados que le confieren significado y sentido. Además, se produce en el marco de una trama de relaciones interpersonales que posee una dimensión sociocultural y personal que depende de los valores, normas, códigos de comunicación y de la historia evolutiva y de relaciones personales (Vargas, 2009). En este sentido, por ejemplo, el estudio del engaño y la mentira como precipitantes de ofensas puede ofrecer contribuciones de interés a la psicología social y de la comunicación (Masip, Garrido, \& Herrero, 2004) y muy específicamente, al estudio de la actitud ante la ofensa o agravio.

\section{Actitudes ante situaciones de agravio}

Para Rokeach (1968), una actitud es una organización de creencias irracionales, relativamente duradera que desencadena una determinada acción con respecto a un objeto o situación. En la psicología social actual, se postulan tres dimensiones de las actitudes: la dirección (estar a favor o en contra), la intensidad o grado y la centralidad dentro del sistema de actitudes del sujeto. Las actitudes ante situaciones de ofensa son predisposiciones personales a responder de una determinada manera cuando se es humillado u objeto de una injuria personal. Se trata de modalidades de comportamiento que reflejan disposiciones del ánimo, del pensamiento y de la voluntad, que se manifiestan en distintas conductas (Moreno \& Pereyra, 2000, 2001; Vargas, 2009).

Este trabajo adopta el modelo de actitudes ante situaciones de agravio (MASA) de Moreno y Pereyra (2000). Estos autores, a partir de su trabajo con el Cuestionario de Actitudes ante Situaciones de Agravio (CASA), postulan la existencia de tres grupos de respuestas frente a la ofensa que a su vez configuran siete modalidades específicas: respuestas pasivas (sometimiento y negación), respuestas agresivas (hostilidad, rencor y venganza) y respuestas prosociales (demanda de explicación y perdón).

Las respuestas pasivas caracterizan a las personas que optan por una actitud conformista o de aceptación de la ofensa. Manifiestan una tendencia al hipercontrol para mantener el equilibrio anterior amenazado por el altercado. En esta dimensión, distinguimos dos modalidades: el sometimiento y la negación. En la actitud de sometimiento prevalece el control emocional, quedando por lo tanto el sujeto inhibido y sin las fuerzas necesarias para una respuesta activa, realizando justificaciones autodescalificadoras, por ejemplo: "me lo merezco" o "es mi culpa". La sobreexigencia y el fuerte sentido del deber suelen ser los factores principales de la sumisión (Vargas, 2009). Moreno \& Pereyra (2000, p. 17) definen la actitud de sometimiento como "la conducta de subordinación del juicio, la decisión o los afectos propios a la actitud del ofensor, haciendo una exclusión consciente del recuerdo, la idea y los sentimientos relacionados con el agravio sufrido, y haciendo un esfuerzo por olvidar el asunto". En la otra modalidad, la negación, prevalece el control perceptivo, distorsionando la representación de la realidad, de modo tal que ignora la situación perturbadora, no llegando a percibirla como una ofensa; se define, por tanto, "como la exclusión de 
la conciencia del hecho y los sentimientos concomitantes relacionados con el acto ofensivo" (Moreno \& Pereyra, 2000, p. 18).

Las respuestas agresivas implican emociones ofensivas hacia el agresor que provocan agresión física y/o verbal o bien resentimiento que predispone a una enemistad duradera. Entre las respuestas agresivas, se encuentran: la hostilidad y el rencor. La primera se define como una "conducta impulsiva, inmediata y reactiva que intenta hacer daño al agresor, acometiendo contra él en el mismo acto de la ofensa" (Moreno \& Pereyra, 2000, p. 19). Se asocia a una amplia variedad de emociones: irritabilidad, enfado, rabia, enojo, ira, cólera, disgusto, cinismo, entre otras (Vargas, 2009). Por otro lado, la actitud de rencor, se define como "el amargo y enraizado recuerdo de una injuria particular de la cual desea uno satisfacerse" (Moreno \& Pereyra, 2000, p. 20). Por último, la venganza, en la que la violencia no tiene función defensiva sino irracional se define como "la búsqueda intencional y planificada del desquite, tratando de proporcionar al ofensor un castigo similar o superior al padecido" (Moreno \& Pereyra, 2000, pp. 20-21). Normalmente, el individuo con una experiencia vital adaptada no siente esa necesidad, aunque haya sido insultado o dañado.

Finalmente, se encuentran las respuestas prosociales, las que van dirigidas a enfrentar el conflicto de manera asertiva y dirigidas tanto a expresar los sentimientos producidos por la ofensa, como a reestablecer en la medida de lo posible la relación con la otra persona, o al menos, a restaurar el daño afectivo causado por la ofensa (Moreno \& Pereyra, 2000). Entre estas respuestas se encuentran la búsqueda de explicación, actitud que lleva a clarificar la discordia afrontando el conflicto, centrándose en el problema en lugar de en la emoción y en el vínculo en lugar de en la represión o en la agresión (Vargas, 2009). La exigencia de una explicación es definida como: "la actitud de demandar al ofensor justificaciones y motivos que den cuenta de su proceder, exigiendo recuperar o reparar, total o parcialmente, el daño ocasionado, como condición necesaria para reparar el vínculo" (Moreno \& Pereyra, 2000, p. 23). En cuanto al perdón o reconciliación, Enright (1994, p. 76) lo ha definido como "la capacidad que un individuo posee para sobrellevar una injusticia y restablecer una relación de amor con los otros". El autor considera el perdón como el estadio más desarrollado de la evolución moral en la personalidad. Es la actitud de cuidar genuinamente el vínculo de afecto hacia el otro, movilizando conductas prosociales orientadas al diálogo y la superación de la discordia. El ofendido va más allá de la aclaración satisfactoria de las causas de la controversia, porque cierra las puertas a las acciones de hostilidad, venganza o rencor. El perdón no es una reacción espontánea, supone un proceso de adquisición y desarrollo de determinadas actitudes y competencias para enfrentar el dolor que comporta una ofensa, sin descalificar o descartar las demás actitudes.

Las actitudes ante situaciones de agravio se ven influidas por el aprendizaje, el modelamiento y las prácticas de crianza de los padres, la creencia religiosa, el clima social familiar, y en general, por las costumbres y valores que promueve el grupo social al que pertenece la persona (Moreno \& Pereyra, 2000; Pereyra, 2003; Rodríguez \& Moreno, 2011; Vargas, 2009).

\section{Actitudes ante el agravio y género}

El estudio metaanalítico de Fehr, Gelfand \& Nag (2010) analizó 175 trabajos para determinar los correlatos interpersonales del perdón. Los resultados mostraron además de la naturaleza multifacética del perdón, que los constructos situacionales explican más varianza que las características de la víctima y que los efectos de variables como el género y la edad son insignificantes. Otro estudio metaanalítico, elaborado por Miller, Worthington \& McDaniel (2008) halló que las mujeres perdonan más que los hombres, y que existen amplias diferencias de género en la actitud de venganza. Se sugieren como influencias moderadoras sobre las diferencias de género las diferencias funcionales en el procesamiento del perdón, en cualidades disposicionales y en indicadores situacionales.

El estudio de Toussaint \& Webb (2005) examina las diferencias de género en los niveles de empatía y perdón y si la asociación entre estos 
dos últimos difiere según el género. Los resultados muestran que las mujeres son más empáticas que los hombres, pero que no hay diferencias de género para el perdón. La empatía estaba asociada con el perdón para los varones, pero no para las mujeres.

Asimismo, distintas investigaciones indican que tanto la represión o negación de la agresividad como la exteriorización violenta de las emociones hostiles, pueden asociarse con graves trastornos de la salud mental y física (Pereyra, 2003; Pereyra \& Agüero de Kerbs, 1998). De ello se infiere que las conductas de diálogo, perdón y reconciliación se corresponderían con una buena salud mental. En la investigación realizada por Pereyra \& Agüero de Kerbs (1998) con una muestra de adultos jóvenes se encontró que aquellos que declaraban tener más síntomas psicosomáticos exhibían mayores puntuaciones en las escalas de venganza y rencor. Por el contrario, las respuestas de perdón/reconciliación correlacionaron negativamente con síntomas de neuroticismo. A su vez, en el estudio de Rijavec, Jurcec \& Mijocevic (2010) se halló que la venganza y la evitación eran predictores significativos de la depresión para los varones, y solo la venganza lo era para las mujeres.

\section{Actitudes ante el agravio y creencia religiosa}

Con relación a la importancia de la creencia religiosa, Pereyra (2003) en un estudio realizado con 863 participantes de diferentes convicciones religiosas procedentes de cinco países americanos encontró diferencias altamente significativas en todas las actitudes ante la ofensa. Los participantes no creyentes obtuvieron mayores puntuaciones en actitudes como la venganza, el rencor y la hostilidad, mientras que los creyentes mostraron mayor disposición para el sometimiento, la negación y los comportamientos que tienden al diálogo (explicación), al perdón y la reconciliación. El estudio de Sheldon \& Honeycutt (2011) sobre el análisis del impacto del género y la religiosidad en la comunicación del perdón encuentra una relación positiva entre religiosidad y estrategias no verbales para expresar el perdón. Por otra parte, este mismo estudio encuentra diferencias de género, siendo los hombres los que emplean las estrategias minimizadoras con mayor frecuencia que las mujeres.

En el contexto español, el trabajo de GarcíaAlandete \& Pérez-Delgado (2005) analiza la relación entre las actitudes religiosas y los valores en jóvenes universitarios, concluyendo que los jóvenes con mayor religiosidad están más preocupados por lograr el respeto de los demás, ser serviciales, obedientes, educados y controlados. Estos valores consideramos que no se corresponderían con actitudes agresivas ante situaciones de ofensa.

\section{Actitudes ante el agravio y clima social familiar}

Con relación al clima social familiar, un estudio realizado por Vargas (2009) con 140 jóvenes entre 18 y 24 años mostró que los jóvenes que perciben un mejor clima familiar están más dispuestos a perdonar. En esta misma línea, el estudio de Pichardo, Fernández \& Azmecua (2002) muestra que los adolescentes cuyo clima familiar es percibido como elevado en cohesión, expresividad, organización, participación en actividades intelectuales y que atribuye importancia a las prácticas y a los valores de tipo ético o religioso, así como con niveles bajos de conflicto, evidencian una mayor adaptación general que sus iguales cuya percepción sobre la familia es por el contrario, de baja cohesión y expresividad o muy elevado conflicto.

Teniendo en cuenta la repercusión que los diferentes tipos de respuesta ante la ofensa tienen en el bienestar personal y en unas adecuadas relaciones interpersonales, los objetivos de este trabajo fueron, en primer lugar, analizar las actitudes de los jóvenes ante las situaciones de agravio que se producen en el contexto familiar en función del género, la creencia religiosa y el clima social familiar y, en segundo lugar, analizar el poder predictivo de estas tres variables sobre las actitudes ante el agravio.

\section{Método}

\section{Participantes}

Los participantes fueron 230 jóvenes, 100 hombres (43.5 \%) y 130 mujeres (56.5\%) con edades com- 
prendidas entre los 18 y los 28 años y un promedio de 21.2 años $(D E=2.9)$. El tipo de muestreo es de carácter incidental. Se pidió el consentimiento informado de jóvenes a partir de 18 años por marcar el punto legal de entrada en la mayoría de edad. La media de edad de los hombres fue de 22.3 (DE $=3.2)$ y la de las mujeres de $20.3(D E=2.4)$. El $81.3 \%(n=187)$ tenía estudios de grado medio, el $10 \%(n=23)$, estudios universitarios y el $8.7 \%(n$ = 20), estudios básicos.

\section{Instrumentos y variables}

Cuestionario de Actitudes ante Situaciones

de Agravio versión Familia (CASA-F)

(Moreno Eु Pereyra, 2000)

El CASA-F consta de 42 ítems y evalúa siete actitudes ante situaciones de ofensa en el ámbito familiar: negación, sometimiento, venganza, rencor, hostilidad, explicación y perdón. Las siete escalas (actitudes) se agrupan en tres factores: Actitudes Pasivas, Agresivas y Prosociales. El primer factor, incluye las escalas de negación y sometimiento y se define como la disposición a interiorizar los impulsos hostiles, reprimiéndolos o negándolos. El segundo agrupa las actitudes de venganza, hostilidad y rencor, las tres manifestaciones de exteriorización de la agresividad ante una situación de ofensa. Y el tercer factor agrupa las actitudes de explicación y perdón, que reflejan la tendencia al uso del diálogo y la negociación a la hora de superar los conflictos interpersonales.

La prueba consiste en seis relatos cortos, cinco específicos del CASA-F en los que se dan situaciones de ofensa en el ámbito familiar y uno del CASA original y que es incorporado como medida de fiabilidad. Los relatos se centran en: 1) relación fraterna, 2) relación paterno-filial, 3) relación de pareja, 4) relación de amistad (relato del CASA original), 5) relación abuelo-nieto y 6) relación de toda la familia con los vecinos. A modo de ejemplo, en el relato 1 la situación de ofensa que se plantea es: "En el momento más difícil de mi vida, mi hermano se negó a darme el apoyo que necesitaba. Por eso...”. Para cada relato, el sujeto debe identificarse con la per- sona ofendida y responder a siete ítems según una escala Likert de cuatro puntos (nunca, casi nunca, frecuentemente y siempre). Cada ítem, identificado con una letra minúscula, representa una actitud ante la ofensa: negación (p. e., relato 1/ítem e: "Trataré de olvidar su proceder"), sometimiento (p. e., relato 1/ítem d: "Aceptaré su proceder porque quizá hice algo que le molestó"), venganza (p. e., relato1/ítem b "Haré lo mismo con él, cuando se encuentre en una situación similar), rencor (p. e., relatol/ítem f: "Tendré en cuenta lo que hizo mientras viva"), hostilidad (p. e., relato1/ítem c: "Le manifestaré todo el dolor y la rabia que me hizo sentir", explicación (p. e., relato1/ítem a: "Le preguntaré por lo que hizo cuando se dé la oportunidad") y perdón (p. e., relatol/ítem g: "Buscaré el momento adecuado para intentar restaurar la relación”).

Teniendo en cuenta que no se dispone de una version adaptada al español que se habla en España, se entregó el Cuestionario CASA-F a dos jueces con la finalidad de evaluar el vocabulario y la redacción de los ítems. Ambos consideraron conveniente adaptar algunas expresiones del lenguaje de alguno de los relatos para hacerlos comprensibles a población española. Por ejemplo, el relato 5: "Mi abuela no se acordó de mi cumpleaños, sin embargo le vive haciendo muchos regalos a mis primos" fue modificado por "Mi abuela no se acordó de mi cumpleaños, sin embargo le hace muchos regalos a mis primos". A su vez, el relato 4: "Un amigo mío, en una reunión social, me humilló magnificando en público los rasgos negativos de mi personalidad, me dejó en ridículo frente a los concurrentes" fue modificado por "Un amigo mío, en una reunión social, me humilló exagerando en público los rasgos negativos de mi personalidad, me dejó en ridículo frente a los asistentes". La redacción de los ítems no fue modificada sustancialmente.

\section{Escala de Clima Social Familiar (FES) de Moos, Moos $\mathbb{E}$ Trickett (1984, adaptada a la población española por TEA publicaciones, 1995)}

En esta investigación, únicamente se han utilizado las tres subescalas de la dimensión relaciones: cohesión, expresividad y conflicto, que evalúan el 
grado de comunicación y libre expresión dentro de la familia y el grado de interacción conflictiva que la caracteriza. Los índices de consistencia interna en la adaptación española (TEA, 1995) son de 0.78 en la subescala cohesión, 0.69 en la de expresividad y 0.75 en la de conflicto. La variable cohesión familiar del FES evalúa el grado en que los miembros de la familia están compenetrados y se ayudan y apoyan entre sí. La variable expresividad evalúa el grado en que se permite y anima a los miembros de la familia a actuar libremente y a expresar directamente sus sentimientos. Por último, la variable conflicto mide el grado en que se expresan libremente y abiertamente la cólera, la agresividad y el conflicto entre los miembros de la familia.

\section{Cuestionario de datos sociodemográficos}

Recoge información sobre las variables edad, nivel de estudios, profesión y creencia religiosa. La variable creencia religiosa se evaluó a partir de la pregunta: "Su creencia religiosa ocupa en la práctica un lugar: importante, poco importante o nada importante". La variable fue operacionalizada en función de la elección realizada por el sujeto.

\section{Resultados}

\section{Análisis preliminares: validez y fiabilidad del CASA-F}

La falta de estudios previos de validación del CASA-F en España supuso plantearnos como objetivo colateral del estudio analizar la validez y fiabilidad del cuestionario en este contexto. Para estudiar la validez de constructo se realizó un análisis de componentes principales con rotación ortogonal (VARIMAX), siguiendo el procedimiento de los autores de referencia (Moreno \& Pereyra, 2000). Se utilizó el paquete estadístico SPSS 19.0 para Windows. El índice aceptable de Kaiser-MeyerOlkin $(\mathrm{KMO}=0.713)$ y la prueba de esfericidad de Bartlett $\left(\chi^{2}(861, N=230)=2768.3 p<\right.$ $0.001)$, indicaron la posibilidad estadística de realizar la factorización de los ítems del cuestionario. Tras el análisis inicial se aplicó el criterio
“Scree Test" de Cattell (1966) para determinar el número de factores por considerar en la posterior rotación. La representación gráfica de los valores propios señaló como solución factorial adecuada la de tres factores, coincidiendo con los estudios previos y los presupuestos del marco teórico. El primer factor (valor propio 5.4) agrupó a la casi totalidad de los ítems de las escalas venganza, rencor y hostilidad, pertenecientes a la dimensión de respuestas agresivas ante situaciones de ofensa, con una varianza común explicada del $11.1 \%$. El segundo factor (valor propio 4.0) agrupó a los ítems de las actitudes prosociales, es decir, explicación y perdón, con una varianza común explicada del $9.5 \%$. Finalmente, el tercer factor (valor propio 2.4) agrupó a los ítems de las escalas sometimiento y negación de las actitudes pasivas, con una varianza explicada del $7.7 \%$. La Tabla 1 resume los ítems del CASA-F y las saturaciones factoriales obtenidas.

La mayoría de los ítems alcanzaron saturaciones superiores o próximas a 0.3 en las escalas a las que fueron asignados en el estudio original de Moreno y Pereyra (2000), apoyándose en su conjunto la estructura factorial del cuestionario CASA-F en jóvenes españoles. Tan solo dos ítems no alcanzaron saturación significativa en las escalas esperadas: los ítems 6a y 6e del factor Actitudes Agresivas. El ítem 6a ("Recordaré cada vez que los vea los momentos que me han hecho pasar") y el ítem 6e ("Intentaré reunir a los vecinos para tomar medidas al respecto") no son entendidos como respuestas agresivas de rencor y hostilidad, respectivamente. El ítem 6a no saturó significativamente en ningún factor, no obstante, la saturación más alta la obtiene en el factor de Actitudes Agresivas (0.101), por lo que consideramos que puede mantenerse en ese factor. Por otro lado, el ítem 6e saturó significativamente en el factor Actitudes Prosociales (0.42) lo que nos lleva a concluir que en nuestro contexto, la redacción de ese ítem no es adecuada para medir hostilidad y es entendido como una actitud de negociación. Se hace necesario reformular su redacción, incidiendo en el aspecto de hostilidad que originalmente pretende evaluar. La propuesta sería: "Intentaré reunir a los vecinos para tomar medidas intimidatorias al respecto". 


\section{TABLA 1}

Saturaciones factoriales de los items del CASA-F agrupados por factores y escalas

\begin{tabular}{|c|c|c|}
\hline Factores & $\begin{array}{c}\text { Escalas } \\
\text { (Actitudes) }\end{array}$ & Saturaciones e ítems \\
\hline \multirow{3}{*}{$\begin{array}{l}\text { 1. Actitudes } \\
\text { Agresivas }\end{array}$} & Venganza & $\begin{array}{l}\text { (0.464) Ítem 1b. Haré lo mismo con él, cuando se encuentre en una situación } \\
\text { similar. } \\
(0.375) \text { Ítem 2a. Le haré sufrir oportunamente por lo que hizo. } \\
(0.419) \text { Ítem 3g. Haré lo mismo cuando tenga la oportunidad. } \\
(0.606) \text { Ítem 4f. Pondré en evidencia sus defectos ante quien más le moleste. } \\
(0.409) \text { Ítem 5e. Haré lo mismo el día de su cumpleaños. } \\
(0.418) \text { Ítem 6d. Pondré música a todo volumen cuando sepa que están durmiendo. }\end{array}$ \\
\hline & Rencor & $\begin{array}{l}\text { (0.611) Ítem 1f. Tendré en cuenta lo que hizo mientras viva. } \\
(0.421) \text { Ítem 2e. Recordaré toda la vida lo que me hizo. } \\
(0.489) \text { Ítem 3d. Tendré en cuenta lo que hizo mientras dure nuestra relación. } \\
(0.475) \text { Ítem 4c. Tendré en cuenta que me humilló cada vez que me encuentre con él. } \\
(0.516) \text { Ítem 5b. Tendré presente esta ofensa mientras ella viva. } \\
(0.101) \text { Ítem 6a. Recordaré cada vez que los vea los momentos que me han hecho } \\
\text { pasar. }\end{array}$ \\
\hline & Hostilidad & $\begin{array}{l}\text { (0.307) Ítem 1c. Le manifestaré todo el dolor y la rabia que me hizo sentir. } \\
(0.592) \text { Ítem } 2 \mathrm{~b} \text {. Me defenderé insultándolo. } \\
(0.301) \text { Ittem 3a. Le diré lo ingrato/a y traicionero/a que fue. } \\
(0.368) \text { Ítem 4g. Inmediatamente le diré todo lo que pienso de él. } \\
(0.364) \text { Ítem } 5 \text { f. Le expresaré todo mi enojo. } \\
(0.013) \text { Ítem 6e. Intentaré reunir a los vecinos para tomar medidas al respecto. }\end{array}$ \\
\hline \multirow{2}{*}{$\begin{array}{l}\text { 2.Actitudes } \\
\text { Prosociales }\end{array}$} & Explicación & $\begin{array}{l}\text { (0.634) Ítem 1a. Le preguntaré por lo que hizo cuando se dé la oportunidad. } \\
(0.619) \text { Ítem } 2 \mathrm{~g} \text {. Le preguntaré, cuando se tranquilice, porqué reaccionó de esta } \\
\text { manera. } \\
(0.337) \text { Ítem } 3 \text { f. Le pediré oportunamente, una explicación sobre su conducta. } \\
(0.565) \text { Ítem 4e. Le pediré una explicación sobre su proceder. } \\
(0.668) \text { Ítem } 5 \text { d. Le preguntaré oportunamente porqué actuó de esa manera. } \\
(0.460) \text { Ítem 6c. Aprovecharé para preguntarles, en una ocasión propicia, si tienen } \\
\text { algún problema con nosotros. }\end{array}$ \\
\hline & Perdón & $\begin{array}{l}\text { (0.338) Ítem 1g. Buscaré el momento adecuado para intentar restaurar la } \\
\text { relación. } \\
(0.484) \text { Ítem } 2 \mathrm{f} \text {. Hablaré con él sobre lo ocurrido para hacer las paces. } \\
(0.204) \text { Item 3e. Dialogaré para buscar una solución que mejore nuestra relación. } \\
(0.528) \text { Item 4a. Buscaré a través del diálogo la forma de reestablecer la amistad. } \\
(0.608) \text { Item 5c. Intentaré que reconsidere su proceder para mejorar la relación. } \\
(0.308) \text { Item 6f. Los invitaré a casa para intentar mejorar la relación. }\end{array}$ \\
\hline \multirow[t]{2}{*}{$\begin{array}{l}\text { 3. Actitudes } \\
\text { Pasivas }\end{array}$} & Sometimiento & $\begin{array}{l}\text { (0.414) Ítem } 1 \mathrm{~d} \text {. Aceptaré su proceder porque quizá hice algo que le molestó. } \\
(0.537) \text { Ítem } 2 \mathrm{c} \text {. Aceptaré su proceder porque los padres tienen autoridad para } \\
\text { reaccionar así. } \\
(0.203) \text { Ítem } 3 \mathrm{~b} \text {. Pensaré que quizá se ha sentido solo/a por mi culpa. } \\
(0.537) \text { Ítem } 4 \mathrm{~d} \text {. Aceptaré su proceder porque a veces a uno le toca ser el centro de } \\
\text { la crítica. } \\
(0.297) \text { Ítem } 5 \mathrm{~g} \text {. Aceptaré lo que me hizo, dado que los ancianos se olvidan de las } \\
\text { cosas. } \\
(0.495) \text { Ítem } 6 \mathrm{~b} \text {. Lo tomaré como una falta de adaptación al barrio, debido al poco } \\
\text { tiempo que llevan en él. }\end{array}$ \\
\hline & Negación & $\begin{array}{l}\text { (0.416) Ítem 1e. Trataré de olvidar su proceder. } \\
(0.668) \text { Ítem } 2 \mathrm{~d} \text {. Trataré de olvidar lo ocurrido. } \\
(0.410) \text { Ítem 3c. Haré como si nada hubiera ocurrido. } \\
(0.555) \text { Ítem } 4 \mathrm{~b} \text {. Trataré de olvidar lo ocurrido. } \\
(0.335) \text { Ítem } 5 \mathrm{a} \text {. Trataré de olvidar lo que me hizo. } \\
(0.454) \text { Ítem 6g. Ignoraré su conducta. }\end{array}$ \\
\hline
\end{tabular}

Fuente: elaboración propia 
En segundo lugar, se realizaron análisis correlacionales con el objetivo de analizar la validez convergente de las escalas del CASA-F. Se obtuvieron correlaciones significativas de tipo positivo entre las actitudes del mismo factor todas ellas compatibles con el marco teórico. Es decir, existe una fuerte relación positiva entre las actitudes de explicación y perdón $(r=0.56, p<0.05)$, ambas de tipo prosocial; entre negación y sometimiento $(r=0.52, p<0.05)$, ambas actitudes pasivas y entre venganza, rencor y hostilidad, las tres de tipo agresivo (Tabla 2). Estas correlaciones indicaron una adecuada validez convergente. Asimismo, se obtuvieron correlaciones significativas de tipo negativo entre actitudes de distintos factores, resultado también congruente con el marco teórico. Por ejemplo, entre las tres actitudes agresivas (venganza, rencor y hostilidad) y las dos actitudes pasivas (sometimiento y negación). Por otro lado, cabe destacar tres resultados: 1) que la actitud de explicación (prosocial) correlacionó positivamente con la de hostilidad (agresiva) $(r=0.39, p<0.01)$, 2), la actitud de perdón (prosocial) correlacionó positivamente con las dos actitudes pasivas, sometimiento $(r=0.19, p<0.01)$ y negación $(r=0.24$, $p<0.01)$ y 3$)$ negativamente con las de venganza $(r$ $=-0.32, p<0.01)$ y rencor $(r=-0.25, p<0.01)$, dos de las Actitudes Agresivas.

Por último, se realizó el análisis de fiabilidad. La Tabla 3 muestra las medias, desviaciones estándar y alfas de Cronbach obtenidas en el estudio de consistencia interna de las escalas del CASA-F. Como puede observarse, se obtuvieron alfas de Cronbach que oscilaron entre 0.48 y 0.68 . Las escalas de so-

TABLA 2

Coeficientes de correlación de Pearson entre las escalas del CASA-F

\begin{tabular}{lcccccc}
\hline \multicolumn{1}{c}{ Escalas } & 1 & 2 & 3 & 4 & 5 & 6 \\
\hline 1. Sometimiento & - & & & & & \\
2. Negación & $0.52^{* *}$ & & & & & \\
3. Venganza & $-0.17^{* *}$ & $-0.33^{* *}$ & & & & \\
4. Rencor & $-0.17^{* *}$ & $-0.43^{* *}$ & $0.48^{* *}$ & & & \\
5. Hostilidad & $-0.14^{*}$ & $-0.27^{* *}$ & $0.36^{* *}$ & $0.33^{* *}$ & & \\
6. Explicación & -0.08 & -0.11 & -0.08 & -0.07 & $0.39 * *$ & \\
7. Perdón & $0.19^{* *}$ & $0.24^{* *}$ & $-0.32^{* *}$ & $-0.25^{* *}$ & 0.1 & $0.56^{* *}$ \\
\hline
\end{tabular}

$* p<0.05 * * p<0.01$

Fuente: elaboración propia

TABLA 3

Medias, desviaciones estándar y alfa de Cronbach de las escalas del CASA-F

\begin{tabular}{lcccc}
\hline \multicolumn{1}{c}{ Escalas CASA-F } & $\mathrm{M}$ & $\mathrm{DE}$ & \multicolumn{2}{c}{ alfa de Cronbach España / México* } \\
\hline Sometimiento & 13.35 & 2.66 & 0.48 & 0.43 \\
Negación & 15.36 & 3.23 & 0.62 & 0.53 \\
Venganza & 9.69 & 2.6 & 0.56 & 0.62 \\
Rencor & 12.9 & 3.26 & 0.68 & 0.68 \\
Hostilidad & 13.62 & 3.08 & 0.52 & 0.75 \\
Explicación & 17.65 & 3.46 & 0.66 & 0.66 \\
Perdón & 17.14 & 2.8 & 0.57 & 0.64 \\
$\quad$ Factores CASA-F & & & & 0.82 \\
\hline Pasivas & 34.78 & 5.56 & 0.76 & 0.79 \\
Agresivas & 36.19 & 6.87 & 0.75 & \\
Prosociales & 28.72 & 5.13 & 0.71 & \\
\hline
\end{tabular}

* alfa de Cronbach en muestra mexicana (Pereyra, 2009).

Fuente: elaboración propia 
metimiento y hostilidad obtuvieron los índices más bajos, 0.48 y 0.52 , respectivamente. Las escalas de rencor y explicación obtuvieron los índices más elevados, 0.68 y 0.66 , respectivamente. Los índices de consistencia interna alcanzaron valores más elevados cuando el análisis se efectuó agrupando las escalas en los tres factores. Así, el factor que agrupa las Actitudes Agresivas obtuvo un alfa de Cronbach de 0.75 , el factor de Actitudes Pasivas de 0.75 y el factor de Actitudes Prosociales de 0.71.

Si comparamos las alfas de Cronbach de nuestra muestra con los de un estudio realizado en México con 415 sujetos adultos con una edad promedio de 38 años (Pereyra, 2009), observamos altos niveles de concordancia. Podemos concluir que los índices de consistencia interna del CASA-F en población española son similares a los obtenidos en estudios previos realizados en otros contextos.

Actitudes ante situaciones de agravio en función del género y la creencia religiosa

Se realizó un MANOVA multifactorial $2 \times 3$ tomando como variables agrupadoras el género y la creencia religiosa (nada importante, poco importante y muy importante) y como variables dependientes las siete actitudes ante situaciones de agravio (Tabla 4). El contraste multivariado mostró diferencias estadísticamente significativas entre hombres y mujeres $\left(\lambda=0.91, F_{(7,209)}=3.13, p<0.01, \eta_{\text {parcial }}^{2}=0.1\right) \mathrm{y}$ entre los grupos de la variable creencia religiosa $(\lambda$ $\left.=0.86, F_{(14,418)}=1.7, p<0.001, \eta_{\text {parcial }}^{2}=0.07\right)$, pero no en la interacción entre ambas variables $(\lambda$ $\left.=0.95, F_{(14,418)}=7.1, p>0.05, \eta_{\text {parcial }}^{2}=0.03\right)$. Respecto al género, los contrastes univariados alcanzaron significación estadística en dos de las actitudes analizadas: venganza $\left(F_{(1,215)}=4.4, p<\right.$ $\left.0.05 ; \eta_{\text {parcial }}^{2}=0.02\right)$ y explicación $\left(F_{(1,220)}=7.5, p\right.$ $\left.<0.01 ; \eta_{\text {parcial }}^{2}=0.03\right)$. Los hombres puntuaron significativamente más alto en la actitud de venganza (hombre: $M=10.2, D E=2.8$; mujer: $M=$ 9.3, $D E=2.4)$ y las mujeres en la actitud de explicación (mujer: $\mathrm{M}=18.3, \mathrm{DE}=3.3$; hombre: $\mathrm{M}=$ $16.8, D E=3.5)$. Respecto a la creencia religiosa, los contrastes

univariados alcanzaron significación en la actitud de rencor $\left(F_{(2,215)}=4.9, p<0.01 ; \eta_{\text {parcial }}^{2}=0.04\right) \mathrm{y}$ de perdón $\left(F_{(2,215)}=9, p<0.001 ; \eta_{\text {parcial }}^{2}=0.08\right)$. Las pruebas de comparación a posteriori de Scheffe indicaron que los jóvenes que no le dieron importancia o poca importancia a la creencia religiosa, puntuaron significativamente más alto en rencor que los que le dan mucha importancia (dif. medias $=1.59, p<0.05$; dif. medias $=1.88, p<0.05$, respectivamente) y los jóvenes que no dieron importancia a la creencia religiosa puntuaron significativamente más bajo en perdón, que los que le dan poca y mucha importancia a la creencia religiosa (dif. medias $=-1.6, p<0.01$; dif. medias $=-1.6, p$ $<0.01$, respectivamente).

\section{TABLA 4}

Comparación de medias entre los grupos de las variables género y creencia religiosa en las actitudes ante situaciones de agravio

\begin{tabular}{|c|c|c|c|c|c|c|c|c|c|}
\hline \multirow[b]{3}{*}{ Actitudes } & \multicolumn{2}{|c|}{ Género } & & & \multicolumn{3}{|c|}{ Importancia de la creencia religiosa } & & \multirow[b]{3}{*}{$\eta^{2}$} \\
\hline & Hombre & Mujer & & & Nada & Poco & Mucho & & \\
\hline & $\mathrm{M}(\mathrm{DE})$ & M (DE) & & $\eta^{2}$ & $M(D E)$ & $M(D E)$ & $M(D E)$ & & \\
\hline Sometimiento & $13.3(2.6)$ & $13.3(2.7)$ & & 0.001 & $12.9(2.7)$ & $13.6(2.6)$ & $13.8(2.4)$ & + & 0.02 \\
\hline Negación & $15.2(3.1)$ & $15.4(3.3)$ & & 0.001 & $15(3.4)$ & $15.3(3.2)$ & $16(2.9)$ & & 0.01 \\
\hline Venganza & $10.2(2.8)$ & $9.3(2.4)$ & $*$ & 0.02 & $10.1(2.9)$ & $9.7(2.6)$ & $8.9(1.8)$ & & 0.03 \\
\hline Rencor & $12.7(3.1)$ & $13.2(3.4)$ & & 0.006 & $13.1(3.3)$ & $13.4(3.3)$ & $11.6(2.8)$ & $* *$ & 0.04 \\
\hline Hostilidad & $13.3(2.8)$ & $13.8(3.3)$ & & 0.005 & $13.5(3.3)$ & $14.1(3.2)$ & $12.7(2.2)$ & + & 0.02 \\
\hline Explicación & $16.8(3.5)$ & $18.3(3.3)$ & $* *$ & 0.034 & $17.1(3.8)$ & $18.2(3.1)$ & $18(3.3)$ & & 0.01 \\
\hline Perdón & $16.9(2.8)$ & $17.3(2.8)$ & & 0.005 & $16.2(3)$ & $17.8(2.4)$ & $17.8(2.6)$ & $* *$ & 0.08 \\
\hline
\end{tabular}

Nota. Hombres: $N=94$. Mujeres: $N=127$. Nada de importancia religiosa: $N=100$. Poca: $N=81$. Mucha: $N=40$. $+p=0.07 * p<0.05 * * p<0.01 . \eta^{2}$ : eta cuadrado parcial.

Fuente: elaboración propia 


\section{Relación entre actitudes ante situaciones de agravio y el clima social familiar}

Previamente al análisis de la relación entre las variables de actitudes ante situaciones de agravio y el clima social familiar percibido, se realizó un análisis descriptivo de estas últimas variables controlando las diferencias en función del género. Se llevó a cabo un MANOVA, tomando como variable agrupadora el género y como variables dependientes, las tres variables del clima social-familiar (cohesión, expresividad y conflicto). El contraste multivariado del MANOVA no mostró diferencias estadísticamente significativas entre hombres y mujeres en la percepción de su clima social familiar $\left(\lambda=0.98, F_{(3,226)}\right.$ $=1.45, p>0.05, \eta_{\text {parcial }}^{2}=0.02$ ).

En segundo lugar, se analizaron las relaciones entre las actitudes ante situaciones de ofensa y las tres variables de clima social familiar mediante análisis correlacionales. Tal y como se observa en la Tabla 5, se obtuvieron correlaciones significativas entre las actitudes prosociales y las variables de clima social familiar: de tipo positivo con cohesión $(r=0.25 ; p<0.01)$ y expresividad $(r=0.26 ; p<$ $0.01)$ y de tipo negativo con conflicto $(r=-0.17 ; p<$ $0.01)$. No se obtuvieron correlaciones significativas entre las Actitudes Pasivas y Agresivas consideradas en su conjunto y las variables de clima social familiar. No obstante, si consideramos las actitudes pasivas y agresivas separadamente, las actitudes de sometimiento $(r=0.15 ; p<0.05)$ y venganza $(r=$ $0.14 ; p<0.05)$ correlacionaron positivamente con la percepción de conflicto familiar y la actitud de rencor correlacionó negativamente con la expresividad familiar $(r=-0.15 ; p<0.05)$.

Capacidad predictiva del género, la creencia religiosa y el clima social familiar sobre las actitudes ante situaciones de agravio

Por último, para analizar la capacidad predictiva del género, la creencia religiosa y el clima social familiar sobre las actitudes prosociales, agresivas y pasivas ante situaciones de ofensa se realizaron tres regresiones jerárquicas múltiples por el método de pasos sucesivos (stepwise). En los tres análisis, el bloque 1 consideraba como variables explicativas el género y la creencia religiosa, mientras que el bloque 2 consideraba las tres variables de clima social familiar (cohesión, expresividad y conflicto). En el primero de los análisis, se obtuvo una correlación múltiple con el criterio de 0.4 y una $R^{2}$ corregida 0.15 en el modelo final $\left(F_{(4,220)}=10.5, p<0.001\right)$. Los resultados de este primer análisis se muestran en la Tabla 6. Se confirmaron como predictores significativos de las actitudes prosociales en el modelo final (paso 4) a las variables creencia religiosa $(\beta=$ $0.19, p<0.011$ ), género $(\beta=0.17, p<0.01)$, expresividad $(\beta=0.27, p<0.001)$ y conflicto familiar $(\beta=-0.15, p<0.05)$. El modelo final explicó un

TABla 5

Coeficientes de correlación de Pearson entre las variables de clima social y las Actitudes ante Situaciones de Agravio

\begin{tabular}{lccc}
\hline & \multicolumn{3}{c}{ Clima social familiar } \\
\cline { 2 - 4 } \multicolumn{1}{c}{ Actitudes } & Cohesión & Expresividad & Conflicto \\
\hline Sometimiento & -0.04 & 0.09 & $0.15^{*}$ \\
Negación & -0.01 & 0.03 & 0.06 \\
Venganza & -0.10 & -0.10 & $0.14^{*}$ \\
Rencor & -0.06 & $-0.15^{*}$ & 0.01 \\
Hostilidad & 0.06 & 0.10 & 0.04 \\
Explicación & $0.24^{* *}$ & $0.20^{* *}$ & $-0.18^{* *}$ \\
Perdón & $0.19^{* *}$ & $0.28^{* *}$ & -0.12 \\
& & & 0.11 \\
Pasivas & -0.03 & 0.07 & 0.07 \\
Agresivas & -0.04 & -0.07 & $-0.17^{* *}$ \\
Prosociales & $0.25^{* *}$ & $0.26^{* *}$ & \\
\hline
\end{tabular}

Nota. ${ }^{*} p<0.05 * * p<0.01$

Fuente: elaboración propia 
$16 \%$ de la varianza del criterio, siendo la variable expresividad familiar la que mayor porcentaje de varianza explica (Paso 3: Cambio $R^{2}=0.08, F_{(3,221)}$ $=11.9, p<0.001$ ).

En el segundo de los análisis, donde se evaluó la capacidad predictiva de los dos bloques de variables sobre las actitudes agresivas, únicamente se introdujo como predictor significativo la creencia religiosa $(\beta=-0.14, p<0.05)$, explicándose tan solo un $1.5 \%$ de la varianza $\left(R^{2}\right.$ corregida $=0.015$,
$\left.F_{(1,223)}=4.47, p<0.05\right)$. Por último, no se confirmó la capacidad predictiva de las variables de género, creencia religiosa y el clima social familiar sobre las actitudes pasivas.

Con el objetivo de profundizar en los resultados relativos a las actitudes prosociales, se realizaron dos nuevos análisis de regresión jerárquica considerando como variables criterio las dos actitudes prosociales, perdón y explicación, respectivamente. En lo que se refiere a la capacidad predictiva sobre la actitud de

TABLA 6

Análisis de regresión múltiple de las variables predictoras de las actitudes prosociales

\begin{tabular}{|c|c|c|c|c|c|}
\hline Variable & B & SEB & $\beta$ & $\mathrm{R}^{2}$ & CambioR $^{2}$ \\
\hline \multicolumn{6}{|c|}{ Variables predictoras del factor Actitudes Prosociales } \\
\hline Paso 1 & & & & 0.04 & $0.04 * *$ \\
\hline Creencia religiosa & 1.4 & 0.49 & 0.19 & & \\
\hline Paso 2 & & & & 0.06 & $0.02 *$ \\
\hline Creencia religiosa & 1.4 & 0.49 & 0.18 & & \\
\hline Género & 1.7 & 0.73 & 0.15 & & \\
\hline Paso 3 & & & & 0.14 & $0.08 * * *$ \\
\hline Creencia religiosa & 1.4 & 0.47 & 0.19 & & \\
\hline Género & 1.9 & 0.7 & 0.17 & & \\
\hline Expresividad & 0.87 & 0.19 & 0.28 & & \\
\hline Paso 4 & & & & 0.16 & $0.02 *$ \\
\hline Creencia religiosa & 1.4 & 0.46 & 0.19 & & \\
\hline Género & 1.9 & 0.7 & 0.17 & & \\
\hline Expresividad & 0.82 & 0.19 & 0.27 & & \\
\hline Conflicto & -0.51 & 0.22 & -0.15 & & \\
\hline \multicolumn{6}{|c|}{ Variables predictoras de la actitud de perdón } \\
\hline Paso 1 & & & & 0.06 & $0.06 * * *$ \\
\hline Creencia religiosa & 0.92 & 0.24 & 0.25 & & \\
\hline Paso 2 & & & & 0.14 & $0.08 * * *$ \\
\hline Creencia religiosa & 0.94 & 0.23 & 0.25 & & \\
\hline Expresividad & 0.44 & 0.1 & 0.29 & & \\
\hline \multicolumn{6}{|c|}{ Variables predictoras de la actitud de explicación } \\
\hline Paso 1 & & & & 0.04 & $0.04 * *$ \\
\hline Género & 1.4 & 0.46 & 0.21 & & \\
\hline Paso 2 & & & & 0.09 & $0.05 * * *$ \\
\hline Género & 1.4 & 0.44 & 0.19 & & \\
\hline Cohesión & 0.37 & 0.1 & 0.23 & & \\
\hline Paso 3 & & & & 0.1 & $0.02 *$ \\
\hline Género & 1.5 & 0.44 & 0.21 & & \\
\hline Cohesión & 0.27 & 0.11 & 0.16 & & \\
\hline Expresividad & 0.28 & 0.13 & 0.15 & & \\
\hline
\end{tabular}

Nota. $* p<0.05 * * p<0.01 * * * p<0.001 N=226$.

Fuente: elaboración propia 
perdón, se constató una correlación múltiple con el criterio de 0.38 y una $R^{2}$ corregida 0.13 en el modelo final $\left(F_{(2,222)}=18.3, p<0.001\right)$. Los resultados se muestran en la Tabla 6 . Se confirmaron como predictores significativos de la actitud de perdón en el modelo final (paso 2) a las variables creencia religiosa $(\beta=0.19, p<0.011)$ y expresividad $(\beta=0.29, p<$ 0.001). La variable creencia religiosa explicó un $6 \%$ de la variabilidad del criterio y la variable expresividad incrementó significativamente el porcentaje de varianza explicada en un $8 \%$. Por último, respecto a la actitud de explicación, se obtuvo una correlación múltiple con el criterio de 0.33 y una $R^{2}$ corregida de 0.1 en el modelo final $\left(F_{(3,223)}=9.3, p<0.001\right)$, introduciéndose como predictores significativos en la ecuación las variables género $(\beta=0.21, p<$ 0.01), cohesión $(\beta=0.16, p<0.05)$ y expresividad $(\beta=0.15, p<0.05)$. La cohesión y la expresividad familiar incrementaron en un $5 \%\left(F_{(2,224)}=11.5\right.$, $p<0.001)$ y en un $2 \%\left(F_{(3,223)}=9.3, p<0.001\right)$, respectivamente, la varianza explicada en el paso 1 por la variable género.

\section{Discusión y conclusiones}

El estudio de las actitudes ante situaciones de agravio en el entorno familiar y el análisis de su relación con el género, la creencia religiosa y el clima social familiar ha sido el eje central de este trabajo. Para ello, en primer lugar, se consideró oportuno comprobar cómo operaban las dimensiones del CASA-F (Moreno \& Pereyra, 2000) en jóvenes españoles. Los resultados en cuanto a validez de constructo y consistencia interna son similares a los de otros contextos estudiados. Los resultados del estudio de consistencia interna del cuestionario han obtenido alfas de Cronbach pobres en algunas escalas, sin embargo, este resultado es similar al obtenido en estudios previos realizados en otros contextos (Canales, 2011; Pereyra, 2009). No obstante, sí que se ha obtenido una adecuada consistencia interna en las escalas de segundo orden (actitudes pasivas, agresivas o prososciales) donde se alcanzan índices superiores a 0.7. Se considera conveniente realizar futuros estudios de validación de este instrumento.
Respecto al estudio de la validez convergente, se ha encontrado una relación significativa entre las escalas del CASA-F, coincidiendo con los resultados de la validación del instrumento en población argentina (Pereyra, 2003) y mexicana (Pereyra, 2009). Así, pues, se relacionan positivamente sometimiento y negación, las dos escalas del factor respuestas pasivas. Igualmente, venganza, hostilidad y rencor, las tres escalas del factor respuestas agresivas y, finalmente, explicación y perdón, las dos escalas del factor respuestas prosociales. Por otro lado, los resultados indican que la actitud de explicación (prosocial) se relaciona positivamente con la de hostilidad (agresiva), pero el perdón se relaciona negativamente con la de venganza y rencor (actitudes agresivas). Este resultado podría interpretarse como que en jóvenes españoles la hostilidad se relaciona con la explicación exigida ante una ofensa, pero una vez se perdona, ya no surgen actitudes agresivas que dificulten la restauración de la relación. No obstante, el perdón se relaciona positivamente con las dos actitudes pasivas (sometimiento y negación), lo que muestra cómo estas actitudes pasivas suelen considerarse socialmente aceptables, y por tanto, se confunden con respuestas asertivas y prosociales como el perdón. Por último, la relación negativa encontrada entre negación y venganza y entre negación y rencor, puede explicarse si se considera la negación como un mecanismo que inhibe la expresión activa de la agresividad.

En segundo lugar, en cuanto a las diferencias entre las actitudes ante situaciones de agravio en función del género, los resultados muestran que los hombres tienden más a ser vengativos y las mujeres optan más por la explicación, hallazgo similar al obtenido por Miller et al. (2008) en su estudio metaanalítico. Este resultado puede ser explicado aludiendo a la tendencia sociocultural de valorar en el hombre las actitudes agresivas, asociadas al mantenimiento de la autoridad y el poder, mientras que en la mujer se potencia una mayor expresión emocional y de ahí su tendencia a pedir explicaciones cuando se siente ofendida. De hecho, en los análisis de regresión el género fue un predictor significativo de las actitudes prosociales, especialmente de la 
actitud de explicación. Un estudio de Rodríguez \& Moreno (2011) sobre las actitudes hacia los roles sexuales concluye que las diferencias entre hombres y mujeres responden a ideales culturales.

En relación con la importancia de la creencia religiosa, se encontró que los jóvenes que no le dan importancia a la creencia religiosa, tienden a actitudes agresivas (venganza, rencor y hostilidad) y en menor medida a actitudes prosociales (explicación y perdón). Este resultado confirma lo obtenido en estudios previos con población argentina (Pereyra, 2003; Vargas, 2009). Por otro lado, en los análisis de regresión se halló que la creencia religiosa tiene poder predictivo sobre las actitudes prosociales, especialmente de la actitud de perdón. Desde la perspectiva ético-moral y teológica, resulta lógico pensar que las personas que se comportan de manera congruente con sus creencias, pueden tender a desarrollar en mayor medida las actitudes prosociales y las competencias para perdonar como la alteridad, la tolerancia con el otro, la empatía, etc., que se promueven en este contexto (Bilbao, Etxebarria, Echano, \& Aguirre, 1999).

Por último, con respecto a la relación entre las actitudes ante situaciones de ofensa y el clima social familiar se obtuvo que la expresividad, la cohesión y el conflicto familiar son predictores de las actitudes prosociales; concretamente, la expresividad familiar predice la actitud de perdón y de explicación y la cohesión familiar predice la actitud de explicación. El conflicto familiar predice de forma negativa las actitudes prosociales en su conjunto. Se confirma, pues, la capacidad predictiva del clima social familiar sobre las actitudes prosociales ante situaciones de ofensa. Nuestro resultado confirma lo obtenido en el estudio de Pichardo et al. (2002), en el que los jóvenes que percibían una alta cohesión y expresividad familiar evidenciaron una mayor adaptación social general comparados con aquelos que percibían baja cohesión, baja expresividad y elevado conflicto en su familia. Otros estudios clásicos como el de Roming \& Bakken (1992 en Martínez, 1997), encontraron que los adolescentes que percibieron sus relaciones familiares con un alto grado de cohesión y adaptación, mostraron más responsabilidad y afecto en sus relaciones interper- sonales. Martínez y Fuertes (1999) señalaron que los hogares que tienen un mejor clima familiar se caracterizan por apoyo, armonía, afecto, cohesión y comunicación abierta, un contexto idóneo para la interacción con los iguales. En general, la mayoría de las investigaciones realizadas sobre el tema, muestran que en los sistemas familiares estables y con un clima familiar afectivo, en el que se presta atención a los estados emocionales y en los que se proporcionan a sus miembros seguridad y afecto, se facilita el desarrollo de actitudes prosociales y, por tanto, se promueve una mejor adaptación social (Jiménez \& López-Zafra, 2011).

Cabe señalar como aportaciones principales de este trabajo, por un lado, el estudio de validez y fiabilidad del cuestionario CASA-F en jóvenes españoles. Este cuestionario permite evaluar un aspecto actitudinal importante de las relaciones afectivas asociado a la resolución de conflictos interpersonales en el ámbito familiar. Por otro lado, los resultados obtenidos ponen de manifiesto diferencias en las actitudes ante la ofensa entre hombres y mujeres, siendo un posible reflejo de pautas educativas y culturales diferentes en función del género. Por último, otra contribución de este trabajo es poner de manifiesto la necesidad de fomentar un clima social familiar adecuado desde la infancia. Los hallazgos indican la importancia de un clima social familiar caracterizado por la expresividad, la cohesión y un bajo conflicto para el adecuado desarrollo afectivo-social de nuestros jóvenes. Este trabajo aporta, pues, claves a un tema que ha despertado recientemente tanto el interés de investigadores como la preocupación de la sociedad en general: la creciente manifestación de conductas agresivas de los jóvenes en el ámbito familiar ante situaciones conflictivas.

Como limitación del estudio, se debe señalar, que la baja varianza explicada en los análisis de regresión pone de manifiesto la necesidad de incorporar otras variables en la explicación de las actitudes ante la ofensa: la calidad del apego adulto, la inteligencia emocional y las habilidades de comunicación que puedan estar asociadas al manejo de las situaciones conflictivas, en los diversos ámbitos de las relaciones interpersonales. 


\section{Referencias}

Bilbao, G., Etxeberría, X., Echano, J., \& Aguirrre, R. (1999). Elperdón en la vida pública. Bilbao, España: Universidad de Deusto, Aula de Ética.

Canales, C. I. (2011). Perfil de la víctima de la violencia conyugal respecto al funcionamiento familiar, la satisfacción marital y las actitudes ante el agravio (Tesis doctoral no publicada). Universidad de Montemorelos, México.

Cattell, R. B. (1966). The Scree Test for the number of factors. Multivariate Behavioral Research, 1(2), $245-276$.

Enright, R. D. (1994). Piaget on the moral development of forgiveness: Identity or Reciprocity? Human Development, 37(2), 63-80.

Fehr, R., Gelfand, M. J., \& Nag, M. (2010). The road to forgiveness: A meta-analytic synthesis of its situacional and disposicional correlates. Psychological Bulletin, 136(5), 894-914. doi: 10.1037/a0019993

García-Alandete, J., \& Pérez-Delgado, E. (2005). Actitudes religiosas y valores en un grupo de jóvenes universitarios españoles. Anales de Psicología, 21(1), 149-169.

Jiménez, M. I., \& López-Zafra, E. (2011). Actitudes sociales y adaptación social en adolescentes españoles: el papel de la inteligencia emocional percibida. Revista de Psicología Social, 26(1), 105-117.

Lafuente, J., \& Cantero, M. J. (2010). Vinculaciones afectivas. Apego, amistad y amor. Madrid: Pirámide.

Martínez, J. L. (1997). Desarrollo personal, ambiente familiar y relaciones de pareja en la adolescencia. Revista de Psicología Social, 12(1), 59-78.

Martínez, J. L., \& Fuertes, A. (1999). Importancia del clima familiar y la experiencia de pareja en las relaciones de amistad adolescentes. Revista de Psicología Social, 14(2-3), 235-250.

Masip, J., Garrido, E., \& Herrero, C. (2004). Definición de engaño. Anales de Psicología, 20(1), 147-171.

Mikulincer, M., \& Shaver, P. R. (2005). Attachment theory and emotions in close relationships: Exploring the attachment-related dynamics of emotional reactions to relational events. Personal Relationships, 12(2), 149-168.

Miller, A. J., Worthington, E. L., \& McDaniel, M. A. (2008). Gender and forgiveness: A meta-analytic review and research agenda. Journal of Social and Clinical Psychology, 27(8), 843-876.

Moos, R. H., Moos, B. S., \& Trickett, E. (1995). Escala de Clima Social-Familiar (FES). Madrid: TEA.

Moreno, J. E., \& Pereyra, M. (2000). Cuestionario de Actitudes frente a Situaciones de Agravio. Entre Ríos, Argentina: Universidad Adventista de la Plata.

Moreno, J. E., \& Pereyra, M. (2001). Attitude toward Offenders Scale: Assessment, validation and research. En M. Martínez (Ed.), Prevention and control of aggression and the impact on its victims (pp. 377-384). Nueva York: Kluwer Academic/Plerum Publishers.

Pereyra, M. (2003). Reconciliación, cómo reparar los vínculos dañados. Montemorelos, México: Publicaciones Universidad de Montemorelos.

Pereyra, M. (junio, 2009). El CASA-F en jóvenes mexicanos. Comunicación presentada en el XXXII Congreso Interamericano de Psicología, Guatemala.

Pereyra, M., \& Agüero de Kerbs, M. (1998). Personalidad, esperanza-desesperanza, control de la agresividad y la salud mental en adventistas y no adventistas. Theologika, 13(2), 330-355.

Pichardo, M. C., Fernández, E., \& Amezcua, J. A. (2002). Importancia del clima social familiar en la adaptación personal y social de los adolescentes. Revista de Psicología General y Aplicada, 55(4), 575-590.

Pietromonaco, P. R., Greenwood, D., \& Feldman, L. (2004). Conflict in adult close relationships: An attachment perspective. En W. S. Rholes \& J. A. Simpson (Eds.), Adult attachment: New directions and emerging issues (pp. 267-299). New York: Guilford.

Rijavec, M., Jurcec, L., \& Mijocevic, I. (2010). Gender differences in the relationship between forgiveness and depression/happiness. Psihologijske Teme, 19(1), 189-202.

Rodríguez, E. S., \& Moreno, J. E. (2011). Actitudes ante situaciones de agravio. Un estudio comparativo entre oficiales de fuerzas de seguridad y estudiantes universitarios. Universitas Psychologica, 10(2), $371-380$.

Rokeach, M. (1968). Beliefs, attitudes, and values: A theory of organization and change. San Francisco: Jossey-Bass. 
Sheldon, P., \& Honeycutt, J. (2011). Impact of gender and religiosity on forgiving communication. Journal of Communication and Religion, 34(1), 59-75.

Toussaint, L., \& Webb, J. R. (2005). Gender differences in the relationship between empathy and forgiveness. Journal Social Psychology, 145(6), 673-685.
Vargas, J. A. (2009). Percepción del clima socio-familiar y actitudes ante situaciones de Agravio en la Adolescencia Tardía. Interdisciplinaria, 26(2), 289-316. 
